\title{
EVALUACIÓN DE HIDROCARBUROS AROMÁTICOS POLICÍCLICOS EN SEDIMENTOS SUPERFICIALES DE LOS ESTEROS EL PAJAL Y CÁNDAMO DE LA BAHÍA DE TUMACO
}

\author{
Robinson Fidel Casanova Rosero ${ }^{1}$ \\ Elkin Darío Castellón-Castrillón ${ }^{2}$ \\ Mónica María Zambrano Ortiz ${ }^{3}$ \\ Grupo de Investigación en Protección del Medio Marino, \\ Centro Control Contaminación del Pacífico (CCCP), \\ Vía El Morro, Capitanía de Puerto, San Andrés de Tumaco, Nariño, Colombia \\ Tél: +57(2) 72726 37, Fax: +57(2) 7271180 \\ e-mail: rcasanova@dimar.mil.co ${ }^{1}$, elkindario80@hotmail.com ${ }^{2}$, monyzambrano@yahoo.com ${ }^{3}$
}

Casanova, R., E. Castellón-Castrillón y M. Zambrano. 2006. Evaluación de hidrocarburos aromáticos policíclicos en sedimentos superficiales de los esteros El Pajal y Cándamo de la bahía de Tumaco. Boletín Científico CCCP, (13): 85-96.

Recibido en julio de 2006; aceptado en diciembre de 2006

\section{RESUMEN}

En este estudio se evaluaron los niveles de 16 hidrocarburos aromáticos policíclicos en sedimentos superficiales recolectados a través de tres monitoreos en seis estaciones de muestreo, ubicadas en los esteros El Pajal y Candamo de la bahía de Tumaco: el primero en mayo de 2005, catorce meses después de ocurrido un derrame de petróleo; el segundo en diciembre de 2005, y el último en abril de 2006. A través del periodo de estudio los niveles de hidrocarburos aromáticos policíclicos oscilaron entre no detectados y $232 \mathrm{ng} / \mathrm{g}$, reflejando una variabilidad espacial notoria, asociada con la cercanía a una fuente de entrada con residuos de petróleo. Por otra parte, los promedios para la sumatoria de los hidrocarburos aromáticos policíclicos detectados mostraron una tendencia temporal decreciente, pasando de $190 \mathrm{ng} / \mathrm{g}$, obtenido para el primer monitoreo, a 74ng/g para el último; dando lugar a inferir a una contaminación temporal y que el área se encontraba en un estado de autodepuración, como producto de un proceso de contaminación. Finalmente, los niveles detectados para cada uno de los hidrocarburos aromáticos policíclicos se clasificaron por debajo los niveles de riesgo establecidos por los estándares de calidad ambiental canadienses.

PALABRAS CLAVE: hidrocarburos aromáticos policíclicos, cromatografía de gases, espectroscopía de masas, sedimentos, materia orgánica, estuario, derrame de petróleo, contaminación marina.

\section{ABSTRACT}

In the present study, the levels of sixteen polycyclic aromatic hydrocarbons (PAH) were determined in superficial sediments collected during three sampling campaigns carried out on May 2005, 14 months after oil spill, on December 2005 and on April 2006, in six stations at the tidelands. The Pajal and Candamo from Tumaco's Bay, impacted by an oil spill occurred in March 2004. During the period of study the mean values of total PAH showed a decreasing trend, from 190ng/g obtained for the first campaign to $76 \mathrm{ng} / \mathrm{g}$ for the last, letting to infer a temporal contamination and the area was in auto depuration state by product of petrogenic contamination processes. Finally, the concentrations for each of the PAH were found below the levels of risk established by the Canadian Environmental Quality Standards.

KEY WORDS: polycyclic aromatic hydrocarbons, gas chromatography/mass spectroscopy, sediments, organic matter, tidelands, marine pollution. 


\section{INTRODUCCIÓN}

Uno de los principales y más conocidos tipos de contaminación marina es el ocasionado por los hidrocarburos del petróleo, el cual puede afectar aguas, sedimentos y organismos. Los efectos causados por este tipo de contaminación se relacionan con la reducción o destrucción de la vida marina, y la reducción parcial o total de la fauna y flora de las playas y zonas costeras. Los efectos tóxicos del petróleo son de difícil evaluación, debido a la amplia gama de compuestos químicos que lo integra.

El petróleo está constituido principalmente por dos clases de hidrocarburos: alifáticos y aromáticos; dentro de estos últimos se encuentran los hidrocarburos aromáticos policíclicos, HAP, que, a su vez, constituyen la fracción más toxica. Su presencia en el ambiente marino se asocia con derrames en operaciones de manejo (cargue, descargue y transporte) del petróleo y sus derivados, pero también con la precipitación atmosférica, transporte a través de ríos y actividades domésticas e industriales, entre otras fuentes.

Los HAP se clasifican de acuerdo al peso molecular en compuestos de bajo peso molecular (2 y 3 anillos), asociados con el petróleo, y de alto peso molecular (4 y 5 anillos) asociados con productos de la combustión. Además, los HAP de origen petrogénico tienen predominio de derivados alquilados, mientras que en los de origen pirolítico (combustión) predominan los parentales. Estos últimos considerados como contaminantes ubicuos, son más resistentes a la biodegradación y se les atribuye un potencial genotóxico, estando clasificados dentro del grupo 2B (posiblemente carcinógeno para el ser humano) de la Agencia Internacional de Investigación sobre el Cáncer (IARC, 1987)

Los HAP son relativamente estables, son constituyentes para el diagnóstico del petróleo y proveen información relacionada con la fuente y el grado de degradación (Page et al., 1995; Bence y Burns, 1995; Douglas et al., 1996), enmarcándose en el tema central de muchos estudios encaminados a evaluar sus efectos adversos sobre los componentes de los ecosistemas afectados; por ejemplo de ecotoxicidad sobre organismos bivalvos - Venerupis pullastra (Franco et al., 2006)

Bajo el panorama anterior, entre los principales problemas de contaminación que afrontan los ecosistemas estuarinos del Pacífico colombiano se encuentra el impacto generado por descargas de sustancias contaminantes provenientes de fuentes terrestres. Es así como se destacan especialmente en las áreas más pobladas (Buenaventura y Tumaco) los vertidos de aguas residuales de origen doméstico, industrial, agrícola y forestal, que generalmente se realizan sin ningún tipo de tratamiento. Asimismo, se tiene la entrada de hidrocarburos asociada al transporte marítimo (buques pesqueros, pequeñas motonaves dedicadas al transporte de mercancía y lanchas) y derrames accidentales durante operaciones de manejo de petróleo y sus derivados. Estas actividades, entre otras, conllevan al aporte de sustancias alóctonas que perturban el equilibrio de producción y consumo de estos ecosistemas.

Tras lo anterior, en el presente estudio se evaluaron los niveles de HAP en sedimentos de los esteros EL Pajal y Cándamo, zonas con alto riesgo frente la contaminación por hidrocarburos, tras el antecedente de un derrame de crudo ocurrido en marzo de 2004 en las instalaciones del Terminal del Oleoducto Trasandino del Distrito Sur de la empresa ECOPETROL S.A.

La matriz ambiental evaluada corresponde a sedimentos, puesto que constituyen un excelente indicador del grado de contaminación para un área determinada, teniendo en cuenta que los contaminantes orgánicos persistentes se adsorben sobre el material en suspensión, que tiende a sedimentarse y finalmente se acumulan en los sedimentos superficiales; constituyéndose así en un testigo confiable de la afectación de un ecosistema. Es importante señalar que en la costa Pacífica colombiana los estudios concernientes a este tipo de contaminantes en matrices ambientales marinas y/o estuarinas son incipientes.

\section{ÁREA DE ESTUDIO}

Entre mayo de 2005 y abril de 2006 se realizaron tres muestreos de sedimentos superficiales en seis estaciones ubicadas en los esteros El Pajal y Cándamo de la bahía de Tumaco, localizada al suroccidente de Colombia sobre la costa Pacífica, delimitada por las latitudes $1^{\circ} 45^{\prime} 0^{\prime \prime}$ y $2^{\circ} 00^{\prime} 0$ " $\mathrm{N}$ y longitudes $78^{\circ} 30^{\prime} 00^{\prime \prime}$ y $78^{\circ} 45^{\prime} 00^{\prime \prime}$ W (Figura 1). Las estaciones se distribuyeron en un área estimada de unas 170 ha y fueron seleccionadas tras haber sido impactadas por un derrame de crudo del tipo 'South Blend', ocurrido el 24 de marzo de 2004; vertiéndose 
50 barriles, de los cuales se estimó que el $20 \%$ entró en contacto con la zona de manglar aledaña, mientras que el $80 \%$ restante quedó confinado en una zona de seguridad y se estimó que el área total impactada fue de 42.84 ha (CCCP, 2004).

Años atrás el área fue permanentemente receptora de la descarga de aguas de decantación que salían de las piscinas de oxidación del Terminal del Oleoducto Transandino de ECOPETROL S.A., en donde se vertían alrededor de 10000 barriles/mes de aguas de desecho, por lo que el lugar donde se ubica la Estación 1 fue catalogado como estación crítica debido a la contaminación por hidrocarburos (Marrugo, 1995). Esto permite reiterar que en el foco donde se ubica la Estación 1 y el espacio adyacente donde se encuentra la Estación 2, se presentó una contaminación crónica por hidrocarburos.

Por otra parte, una de las características principales del área la representa el tipo de vegetación 'bosque de manglar', que se constituye en una de las fuentes productoras de materia orgánica que es incorporada a los sedimentos. Asimismo, el área superficial del sedimento queda totalmente descubierta durante el período de marea baja, conllevando a la mayor oxigenación del mismo por el contacto directo entre las interfases atmósferasedimento; como también es el periodo donde se presenta escurrimiento y flujo de material mezclado desde el interior de estos esteros hacia la boca de los mismos. En la fase de marea subiendo el proceso es inverso, trayendo como resultado variaciones en las condiciones biofísicas y químicas del área.

\section{METODOLOGÍA}

\section{Reactivos}

Los solventes usados en el análisis de hidrocarburos tales como hexano, metanol, diclorometano, isooctano fueron marca Merck.

La sílica (70-230 mesh) y el sulfato de sodio anhidro de marcas J.T. Baker y Carlo Erba, respectivamente. Para su uso y previamente al tratamiento térmico $\left(170^{\circ} \mathrm{C}\right.$ por 12 horas) fueron sometidos a una limpieza a través de extracción en soxhlet durante ocho horas, utilizando una mezcla hexano-diclorometano (1: 1)

Los estándares individuales correspondientes a naftaleno (99.9\%), acenaftileno (99.9\%), acenafteno (99.9\%), fluoreno (98.4\%), fenantreno $(99.8 \%)$, antraceno (99.9\%), fluoranteno (94.5\%), pireno $(92.2 \%)$, benzo[ $a$ ]antraceno $(99.6 \%)$, criseno $(97.4 \%)$, benzo[ $b$ ]fluoranteno(99.9\%), benzo[k]fluoranteno (99.9\%), benzo[a]pireno (96.4\%), benzo[ghi]perileno (99.1\%), dibenzo[ah]antraceno (99.9\%), indeno[1,2,3-cd]pireno $(99.9 \%)$, surrogates deuterados acenaphthylene- $d_{10}$ (99.9\%), fluorantene- $d_{10}(98.7 \%)$ y perylene- $d_{12}(99.7 \%)$ fueron estándares certificados marca Supelco a los de pureza menor del $99 \%$.

En lo concerniente al estándar interno y al material de referencia (MR) de sedimento, fueron suministrados por el Laboratorio de Estudios Ambientales Marinos de la Agencia Internacional de Energía Atómica de Mónaco (IAEA - MEL)

\section{Muestreo}

Los monitoreos se realizaron: el 17 de mayo (14 meses después de ocurrido un derrame); el primero de diciembre de 2005; y el 27 de abril de 2006. Las muestras de sedimentos se recolectaron con un corazonador (separando los primeros $3 \mathrm{a} 4 \mathrm{~cm}$ superficiales), protegiéndolas con papel de aluminio dentro de bolsas Ziplock, rotuladas, refrigeradas con hielo en una caja Iglood, transportadas al laboratorio y preservadas a $-20^{\circ} \mathrm{C}$ hasta el análisis.

\section{Procedimiento químico}

Aproximadamente $5 \mathrm{~g}$ de sedimento liofilizado y tamizado a través de una malla de 250 um fueron fortificados con 500uL de una mezcla $(1 \mu \mathrm{g} / \mathrm{mL})$ de surrogates (acenaftileno $d_{10}$, fluoranteno- $d_{10}, y$ perileno- $d_{12}$ ), y digeridos con $100 \mathrm{~mL}$ de solución de $\mathrm{KOH}$ metanólica al $3 \% \mathrm{p} / \mathrm{v}$ durante hora y media. Posteriormente, se enfrió, decantó y separó la fase alcohólica para extraerse con $25 \mathrm{~mL}$ de $\mathrm{n}$-hexano por dos veces. El extracto $(1.5 \mathrm{~mL})$ concentrado, a través de un rotaevaporador, fue fraccionado a través de cromatografía de adsorción en una columna de vidrio $(45 \times 1 \mathrm{~cm})$ rellena con $8 \mathrm{~g}$ de sílica desactivada con agua $(5 \% \mathrm{p} / \mathrm{p})$. La parte superior de la columna fue sellada con $1 \mathrm{~cm}$ de sulfato de sodio anhidro, eluyéndose primeramente con $20 \mathrm{~mL}$ de $\mathrm{n}$-hexano (fracción alifática), seguido de $50 \mathrm{~mL}$ de una mezcla de $n$-hexano:diclorometano (1:1) y, finalmente, se eluyó con $20 \mathrm{~mL}$ de diclorometano. Las dos últimas eluciones fueron combinadas, conformando la fracción aromática concentrada en rotavapor hasta $3 \mathrm{~mL}$, luego con corriente suave de nitrógeno hasta 
próximo a sequedad, e intercambiándose el solvente (hexano) a una solución de diclorometano-isooctano (1:4) y dejándose en 50uL; momento donde se fortificó con 50uL de una solución de 4,4'dibromobifenilo de $10 \mu \mathrm{g} / \mathrm{mL}$ (utilizando la solución de restitución como solvente) como estándar interno (SI), el cual no interfiere con ninguno de los analitos de interés; obteniéndose un volumen final de $100 \mu \mathrm{L}$, para analizarse a través de cromatografía de gasesespectrometría de masas (CG-EM)

La cuantificación se realizó a través de un sistema de CG-EM, conformado por las siguientes unidades: inyector 7683 Agilent Technologies; cromatógrafo de gases $6890 \mathrm{~N}$ Network Agilent Technologies; detector selectivo de masas 5973 Network Agilent Technologies; automuestreador 7683 Agilent Technologies.

Las condiciones cromatográficas para el análisis de HAP fueron adoptadas según la literatura (Riezve et al., 1994) así: columna capilar HP5-MS: $30 \mathrm{~m} \times 250 \mu \mathrm{m} \times 0.25 \mu \mathrm{m}$; temperatura del inyector a $300^{\circ} \mathrm{C}$; modo de inyección sin división (Splitless); programación de temperatura del horno, temperatura inicial: $40^{\circ} \mathrm{C}$ durante un minuto; luego fue programada a una rampa de $25^{\circ} \mathrm{C} / \mathrm{min}$ hasta $140^{\circ} \mathrm{C}$ y finalmente a una velocidad de $10^{\circ} \mathrm{C} / \mathrm{min}$ hasta $290^{\circ} \mathrm{C}$, donde se mantuvo durante dos minutos; el volumen de inyección fue $1 \mu \mathrm{L}$; el flujo de gas de arrastre (helio) fue de $1 \mathrm{~mL} / \mathrm{min}$ ); modo de ionización por impacto de electrones (a $70 \mathrm{eV}$ ); modo de identificación monitoreo de ión selectivo (SIM) en el rango de masas desde 50 a 350uma; tiempo para la eliminación del solvente $5 \mathrm{~min}$; amplitud de barrido: $0.2 \mathrm{~s} /$ barrido; las temperaturas de la línea de transferencia y de la fuente de iones fueron de $285^{\circ} \mathrm{C}$ y $230^{\circ} \mathrm{C}$, respectivamente. Calibrándose automáticamente con perfluorterbutilamina, PFTB. Finalmente las señales fueron procesadas en el software Chem Station.

La exactitud determinada con base en el porcentaje de recuperación, para una serie de ensayos con una muestra natural tratada y fortificada con cantidades conocidas de los 16 analitos de interés, osciló entre 58\% para el acenaftileno y $118 \%$ para el benzo[b]fluoranteno; exceptuando el naftaleno, para el cual fue baja, con el 36\%. La precisión con base a la desviación estándar relativa del mismo ensayo osciló entre 3 y $20 \%$. Lo que permitió determinar y clasificar dentro del rango de valores esperados (Tabla I) de un material de referencia (MR), suministrado por el Laboratorio de la Agencia Internacional de Energía Atómica (IAEA-408). Los límites de detección estimados para los compuestos estudiados fueron, en general, menores a $1.0 \mathrm{ng} / \mathrm{g}$.

El método aplicado para el análisis de materia orgánica en sedimentos en la fracción menor a $425 \mu \mathrm{m}$, fue el método de oxidación con dicromato propuesto por Walkley y Black (1934); eliminando las interferencias por cloruros, a través de una serie de lavados con agua destilada, hasta que no se observó turbidez alguna tras la prueba del agua de lavado con una gota de solución de nitrato de plata.

\section{RESULTADOS Y DISCUSIÓN}

Partiendo del criterio de que los sedimentos son buenos indicadores del grado de contaminación de los ecosistemas marinos y estuarinos, puesto que se constituyen en el destino final de los contaminantes que llegan a estos ambientes; los sedimentos recolectados y analizados en las seis estaciones se caracterizaron por presentar en su composición índices arenosos (IA) que fueron desde lodosos $(I A \leq 10 \%)$, pasando por lodoarenosos (IA: 10\%-50\%), hasta areno-lodosos (IA: 50\%-90\%), lo cual favorece la persistencia y la baja movilidad de sustancias contaminantes, entre ellas los HAP.

El contenido promedio de materia orgánica a través de los tres monitores en los sedimentos osciló alrededor de un $12 \%$ y su rango estuvo entre 3.6 y $23 \%$ (Tabla I); en donde los valores para las estaciones 2 , 4, 5 y 6 fueron en general similares, lo que permitiría inferir que la distribución de los HAP en el medio fuera homogénea y conllevaría a que sus concentraciones también fueran similares. Sin embargo, ello no se observó, debido a que el factor gobernante es la acción directa de fuentes externas, que induce a establecer que las estaciones 1 y 2 fueron afectadas por vertido de residuos de petróleo; asociándose con los compuestos de alto peso molecular, de allí la variabilidad espacial encontrada.

Aparte de ello, los HAP pueden ser removidos o transformados en otros compuestos como consecuencia de procesos relacionados con la volatilización, fotooxidación y biodegradación. Para este último se conoce que la velocidad de degradación microbiológica de los HAP en el medio está determinada por varios factores entre ellos: el oxígeno, contenido de nutrientes, temperatura, $\mathrm{pH}$ y salinidad, tal como lo expresan varios autores (Atlas, 1981; Hambrick et 
al.,1980; Kerr y Capone 1988; Zaidi e Imam, 1999). Y se cree que estos parámetros no sean una limitante en el área en dicho proceso, puesto que estudios realizados con cepas bacterianas que se aislaron en la Estación 1 mostraron una alta eficiencia en la transformación del contenido de hidrocarburos aromáticos totales presentes en un crudo, con relación a otros aislados bacterianos de otros puntos de la bahía de Tumaco (Guevara y Cárdenas, 1999)

Durante el primer muestreo no se detectaron naftaleno, acenaftileno, fluoreno antraceno y acenafteno, excepto este último en la Estación 3 con $2.2 \mathrm{ng} / \mathrm{g}$. Esto puede ser una consecuencia de las propiedades relativas relacionadas con su bajo peso molecular, solubilidad y alta volatilidad dentro del grupo de los HAP. Tampoco puede descartarse la degradación por bacterias que pudo desarrollarse y actuar sobre estos hidrocarburos más fáciles de degradar y favorecerse, debido a que los sedimentos están periódicamente oxigenados y nutridos por los recambios continuos de masas de agua que bañan el área a través de los recambios mareales.

Por el contrario, los niveles más altos detectados para este muestreo se registraron en las estaciones 1 y 2 , obteniéndose para el criseno las concentraciones más altas con 232 y 186 ng/g, respectivamente (Tabla I); siguiendo en magnitud el benzo[a]pireno con $152 \mathrm{ng} / \mathrm{g}$, en la Estación 1 . Ello puede ser una consecuencia de los residuos remanentes antiguos de las aguas que se vertieron desde las piscinas de oxidación y/o al derrame ocurrido en marzo del 2004.

La sumatoria para los $16 \mathrm{HAP}(\Sigma \mathrm{HAP})$ durante este muestreo osciló entre $8.0 \mathrm{ng} / \mathrm{g}$ determinado para la Estación 3, hasta 597ng/g para la Estación 1; siendo este último valor 2.6 veces mayor con relación a la sumatoria de $233 \mathrm{ng} / \mathrm{g}$ detectado en la Estación 2; marcando un patrón definido de disminución espacial en el área. En la Estación 5, ubicada frente a una estación de bombeo de piscinas acuícolas, sólo se detectó benzo[a]antraceno con un valor de 34ng/g (Tabla I)

Para el segundo muestreo se presentó una reducción notoria de las concentraciones de los HAP con relación al primero, específicamente en las estaciones 1 y 2 , pero manteniéndose con los más altos niveles con relación a los otros puntos. En las estaciones 3 y 5 se observó un comportamiento inverso; pasando la $\mathrm{SHAP}$ de 8.0 a $90 \mathrm{ng} / \mathrm{g}$, y de 34 a $91 \mathrm{ng} / \mathrm{g}$, respectivamente, siendo similares a la sumatoria determinada para las estaciones 4 (100 ng/g) y $6(83 \mathrm{ng} / \mathrm{g})$ (Tabla I)
Las concentraciones durante este muestreo para el acenafteno, fenantreno, pireno, benzo[ $k]$ fluoranteno e indeno[1,2,3-cd]pireno en las estaciones de estudio fueron similares; con promedios de $2.0,12,5.6$ y $1.9 \mathrm{ng} / \mathrm{g}$, respectivamente.

Durante el tercer muestreo el nivel más alto detectado fue en la Estación 2 para el benzo[a]pireno con $71 \mathrm{ng} / \mathrm{g}$, siguiendo la Estación 1 con un valor de $63 \mathrm{ng} / \mathrm{g}$ para el fenantreno. Las concentraciones concernientes a la sumatoria de los 16 HAP para las estaciones 1 y 2 , indicaron un comportamiento similar al observado durante los dos muestreos anteriores, pero con una disminución notoria, de forma que el nivel promedio de la $\Sigma$ HAP disminuyó desde $190 \mathrm{ng} / \mathrm{g}$, detectado en el primer muestreo, a 74ng/g para el último. De forma similar se presentó esta tendencia en las estaciones 3,4 y 5 en comparación con el penúltimo muestreo realizado. Esto permite inferir que durante el lapso de estudio el área aún estaba atravesando por un período de transición, relacionado con un proceso de autodepuración; como posible consecuencia del derrame ocurrido o debido a una combinación de una contaminación anterior.

También se determinó una relación de aproximadamente tres veces entre la $\Sigma$ HAP calculada para la Estación 5 con la Estación 3, y de aproximadamente dos veces para las estaciones 4 y 6 con relación a la Estación 3; lo que puede asociarse con la combinación de factores tales como las características de los sedimentos (IA=69\%), al bajo contenido de materia orgánica (6.8\%) e influencia de fuentes externas relativamente lejanas en esta última estación.

El comportamiento general de las concentraciones representado en los promedios globales a través de los tres monitoreos, según el número de anillos bencénicos, fue $4>5>3$ con valores de 26,14 y $5.8 \mathrm{ng} / \mathrm{g}$, respectivamente; reflejando que los sedimentos tienen preferencia de absorción por los compuestos de alto peso molecular, como puede corroborarse en la literatura (Page et al., 1999)

Por otra parte, durante este muestreo la relación de concentraciones correspondientes a los compuestos de más de tres anillos con la suma de los HAP de dos y tres anillos fue de 3.5 veces. Relaciones análogas se obtuvieron para los monitoreos anteriores, reiterando la afinidad de los sedimentos por estas sustancias. Estudios similares realizados en muchos países, específicamente en áreas industrializadas, puertos y terminales portuarios, han reportado altas concentraciones de HAP que superan en varios órdenes 
de magnitud a las encontradas en este estudio. Tal como en Lazaret Bay, sobre la costa mediterránea, con niveles para la $\Sigma$ HAP cuyo rango fue desde 1600 a $48000 \mathrm{ng} / \mathrm{g}$ (Benlahcen et al., 1997)

Sin embargo, es importante resaltar que a pesar de que los niveles detectados se encuentran por debajo de los valores de referencia, pueden constituir una ruta de entrada de los HAP hacia organismos tales como bivalvos y crustáceos, entre otros; puesto que estos compuestos presentan altos factores de bioacumulación biota-sedimento (Ariese et al., 1993)

Es así como en otras regiones del mundo se han desarrollado estudios encaminados a determinar los HAP en sedimentos, como es el caso del golfo de Arabia, donde los diferentes países de la región reportaron en 1991 los siguientes niveles: Kuwait, $30.4 \mathrm{ng} / \mathrm{gdw}$; Arabia Saudita, 36.4-761ng/gdw; Bahrain, 47.5-97.5ng/kg dw; UAE, 10.9-21.8ng/gdw, y Oman, 4.5-36.5ng/g dw. Sin embargo, allí se reportaron puntos de alta contaminación en el área costera receptora de efluentes industriales donde los niveles oscilaron desde 5.6 hasta $1334 \mathrm{ng} / \mathrm{gdw}$ (Global_Report, 2003). Witt (1999) también reportó concentraciones individuales de HAP que oscilan entre $2.6 \mathrm{ng} / \mathrm{g}$ y $385 \mathrm{ng} / \mathrm{g}$, en sedimentos superficiales del mar Báltico, rango en el cual se incluyen los valores determinados en el presente estudio. Igualmente determinó un rango comprendido entre 721 y $1871 \mathrm{ng} / \mathrm{g}$ para la sumatoria de 15 de los 16 compuestos tratados en el presente estudio. Asimismo, niveles por debajo de $100 \mathrm{ng} / \mathrm{g}$ son catalogados como niveles bajos de contaminación (Tolosa et al., 2004)

Al final del estudio, en todas las estaciones de muestreo las concentraciones detectadas para el naftaleno, acenaftileno, acenafteno, fluoreno, fenantreno, antraceno, fluoranteno, benzo[ $a]$ antraceno, criseno y benzo[ $a]$ pireno estuvieron por debajo de los criterios de calidad establecidos según la norma canadiense; los cuales comprenden niveles que oscilan entre los 5 y $113 \mathrm{ng} / \mathrm{g}$ (CCME, 1999), dependiendo del tipo de compuesto (Tabla I). El dibenzo[ah]antraceno registrado en la Estación 1 fue la excepción a este comportamiento, pues para este compuesto la norma establece un valor de $6.2 \mathrm{ng} / \mathrm{g}$ y el valor detectado fue de $6.4 \mathrm{ng} / \mathrm{g}$. Igualmente, los niveles para este grupo de sustancias son aún bajos con relación al criterio $(3900 \mathrm{ng} / \mathrm{g})$ calificado por la NOAA como concentración alta para 18 HAP (NOAA, 1990); donde se encuentran 11 de los 16 compuestos de nuestro interés, teniéndose que esta concentración supera en más de 20 veces los valores más altos detectados en el último muestreo en las estaciones 1 y 2 (145 y 128 ng/g, respectivamente). Esto conlleva a concretar que en el área de estudio, específicamente en los esteros de El Pajal y Cándamo, se presentan unos niveles de HAP que van desde niveles bajos a moderados.

Por otra parte, se observó que las muestras provenientes de las estaciones 1 y 2 presentaban un avanzado proceso de degradación de los hidrocarburos, por la escasa presencia de picos resueltos en los cromatogramas frente a la gran envolvente, debida a la mezcla compleja de hidrocarburos no resueltos (UCM) (Figura 2), quedando por definir si el proceso de envejecimiento tiene lugar en el sedimento o estaba ya presente en los aportes originales; debido a una contaminación por petróleo tras obtener un valor de 14 para la relación promedia total de las concentraciones fenantreno/antraceno, considerando que relaciones superiores a 10 se deben a este tipo de fuente (Benlahcen et al., 1997; Baumard et al.,1998)

Finalmente, cabe indicar que sería importante realizar un estudio relacionado con la caracterización de bacterias hidrocarburolíticas, puesto que es posible que éste sea un proceso significativo de la transformación de este tipo de compuestos en el área y se constituyan en una alternativa de aplicación en el campo de la biorremediación de zonas impactadas por derrames de petróleo.

\section{CONCLUSIONES}

- Se observó una disminución significativa en la sumatoria de los HAP a través del período de estudio.

- Los sedimentos en el área mostraron una afinidad de absorción y retención de los HAP de alto peso molecular.

- Se evidencia un avanzado proceso de degradación de residuos de petróleo en la Estación 1.

- Las concentraciones de los HAP representan unos niveles que se califican entre el grado bajo a moderado.

- Los valores detectados en sedimentos, en general, se encuentran por debajo de los niveles estándares de calidad admisibles establecidos por las directrices canadienses para la protección de la vida acuática. 


\section{LITERATURA CITADA}

Ariese, F., S. Kok, M. Verkaik, C. Gooijer, N. Velthorst y J. Hofstraat. 1993. Synchronous fluorescence spectrometry of fish bile: a rapid screening method for the biomonitoring of $\mathrm{PAH}$ exposure. Aquat. Toxicol, (26): 273-286.

Atlas, R. 1981. Microbial degradation of petroleum hydrocarbons: an environmental perspective. Microbiol. Rev, (45): 180-209.

Baker, J. y S. Eisenreich. 1990. Concentrations and fluxes of polycyclic aromatic hydrocarbons and polychlorinated biphenyls across the air-water interface of Lake Superior. Environ. Sci.Technol, (24): 342-352.

Baumard, P., H. Budzinski, Q. Mchin, P. Garrigues, T. Burgeot y J. Bellocq. 1998. Origin and bioavailability of PAHs in the Mediterranean Sea from mussel and sediment records. Estuarine,Coastal and Shelf Science, (47): 77-90.

Bence, A. y W. Burns. 1995. Fingerprinting hydrocarbons in biological resources of the Exxon Valdez spill area. In: Wells, P., J. Butler y J. Hughes. (Eds.).

Benlahcen, K., A. Chaoui, H. Budzinski, J. Bellocq y P. Garrigues. 1997. Distribution and sources of polycyclic aromatic hydrocarbons in some Mediterranean coastal sediments. Marine Pollution Bulletin, (34): 298-305.

Centro Control Contaminación del Pacífico. 2004. Condiciones fisicoquímicas, biológicas y ambientales de la zona afectada por el derrame de crudo ocurrido en el Terminal de ECOPETROL el 25 de marzo de. Tumaco - Nariño. Informe técnico.

Chanbasha, B., P. Jeffrey y L. Hian. 2003. Persistent Organic Pollutants in Singapore's Coastal Marine Environment: Part II, Sediments Water, Air \& Soil Pollution Volume 149, No. 1-4, pp. 315 - 325.

Douglas, G., A. Bence, R. Prince, S. Mcmillen y E. Butler. 1996. Environmental stability of selected petroleum hydrocarbon source and weathering ratios. Environ. Sci. Technol, (30): 2332-2339.

Exxon Valdez Oil Spill: Fate and Effects in Alaskan Waters. ASTM Spec. Tech. Pub. No. 1219. American Society for Testing and Materials, Philadelphia, PA, pp. 161-175.

Franco, M., L. Viñas, J. Soriano, D. De Armas, J. González, R. Beiras, N. Salas, J. Bayona y J. Albaigés. 2006. Spatial distribution and ecotoxicity of petroleum hydrocarbons in sediments from the Galicia continental shelf (NW Spain) after the Prestige oil spill. Marine Pollution Bulletin, (53): 260-271.

Global Report. 2003. Regionally Based Assessment of Persistent Toxic Substances En: http: / / www. chem. unep.ch/pts/gr/Global_Report.pdf

Guevara, L., y B. Cárdenas. 1999. Aislamiento y diferenciación de bacterias marinas degradadoras de petróleo pertenecientes al género pseudomonas en la ensenada de Tumaco (Nariño). Pasto. Tesis (Biología con énfasis en Microbiología Industrial). Universidad de Nariño.

Hambrick, G., R. Delaune y W. Patrick. 1980. Effect of estuarine sediment $\mathrm{pH}$ and oxidation-reduction potential on microbial hydrocarbon degradation. Appl. Environ. Microbiol, (40): 365-369.

IARC, 1987. Overall evaluations of carcinogenicity -an updating of IARC monographs volumes 1 to 42. International Agency for Research on Cancer, Monographs on the Evaluation of Carcinogenic Risks to Humans, Suppl. 7. IARC, Lyon. Francia.

Kerr, R. y D. Capone. 1988. The effect of salinity on the microbial mineralization of two polycyclic aromatic hydrocarbons in estuarine sediments. Mar. Environ. Res. (26): 181-198.

Marrugo, A. 1995. Estudio de la contaminación marina por hidrocarburos en áreas críticas de la costa Pacífica colombiana. Boletín Científico. CCCP (5): 121-145.

Nagpal, N. 1993. Ambient Water Quality Criteria for Polycyclic Aromatic Hydrocarbons (PAHs). Water Quality Branch, Water Management Division, BC Ministry of Environment, Lands and Parks. 177 pp.

Page, D., P. Boehm, G. Douglas, G. y E. Bence. 1995. Identification of hydrocarbon sources in the benthic sediments of the Prince William Sound and 
the Gulf of Alaska following the Exxon Valdez oil spill. In: Wells, P.G., Butler, J.N., Hughes, J.S. (Eds.), Exxon Valdez Oil Spill: Fate and Effects in Alaskan Waters. ASTM Spec. Tech. Pub. No. 1219. American Society for Testing and Materials, Philadelphia, PA, pp. 81-140.

Page, D., P. Boehm, G. Douglas, A. Bence, W. Burns y P. Mankiewiez. 1999. Pyrogenic Polycyclic Aromatic Hydrocarbons in sediments record past human activity: A case study in Prince William Sound, Alaska. Marine Pollution Bulletin. 38, 247-260.

Riezve, S., S. Pat y S. Calus. 1994. Water Análisis. Organic micropollutants. Polyciclic aromatic hydrocarbons. Chaper 4, 117, 118p.
Tolosa, I., S. De Mora, M. Sheikholeslami, J. Villeneuve, J. Bartocci y C. Cattini. 2004. Aliphatic and aromatic hydrocarbons in coastal Caspian Sea sediments. Marine Pollution Bulletin, (48): 44-60.

Walkley y Black. 1934. BS 1377:1975, Test 8.

Witt, G. 1999. Polycyclic Aromatic Hydrocarbons in water and sediment of Baltic Sea. Marine Pollution Bulletin. 31 (4-12), 237-248.

Zaidi, B. y S. Imam. 1999. Factors affecting microbial degradation of polycyclic aromatic hydrocarbon phenanthrene in the Caribbean coastal water. Mar. Poll. Bull. 38(8): 737-742.

FIGURAS Y TABLAS

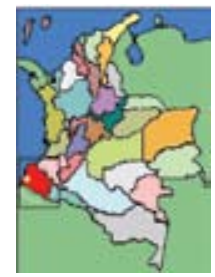

Colombia



Nariño

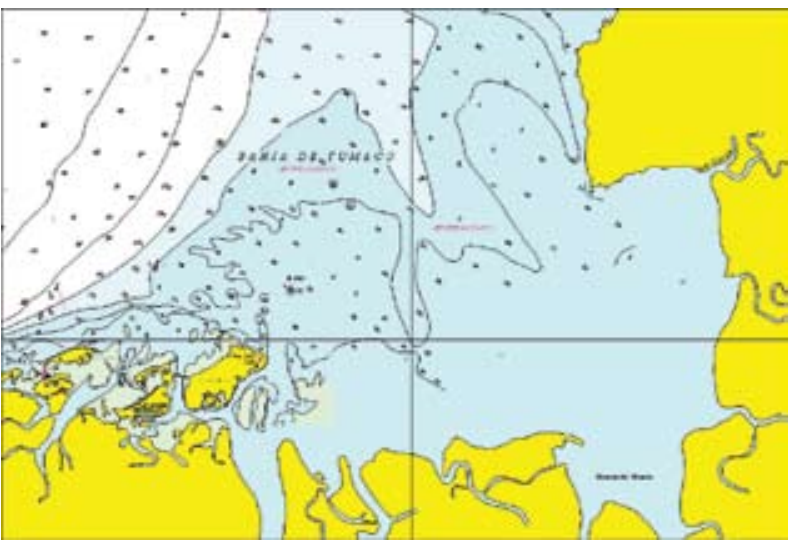

Bahía de Tumaco

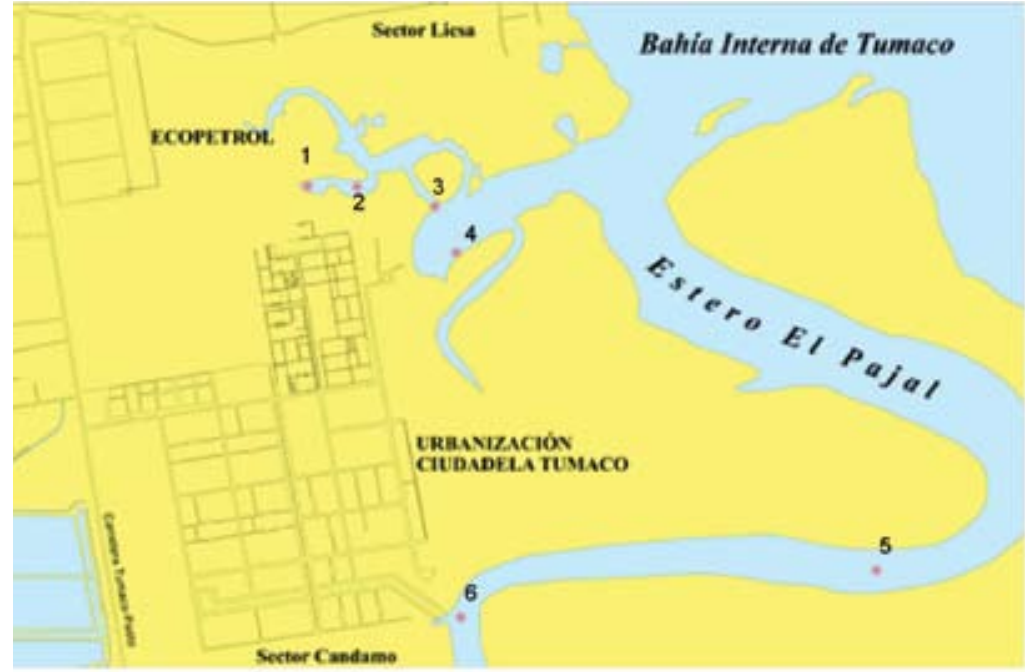

Figura 1. Área de estudio. Ubicación de las estaciones de muestreo. 




Figura 2. Cromatograma correspondiente a la fracción aromática de sedimentos del tercer muestreo de la estación 2. Nota. Los picos definidos y con alta abundancia corresponden a surrogates y estándar interno. 

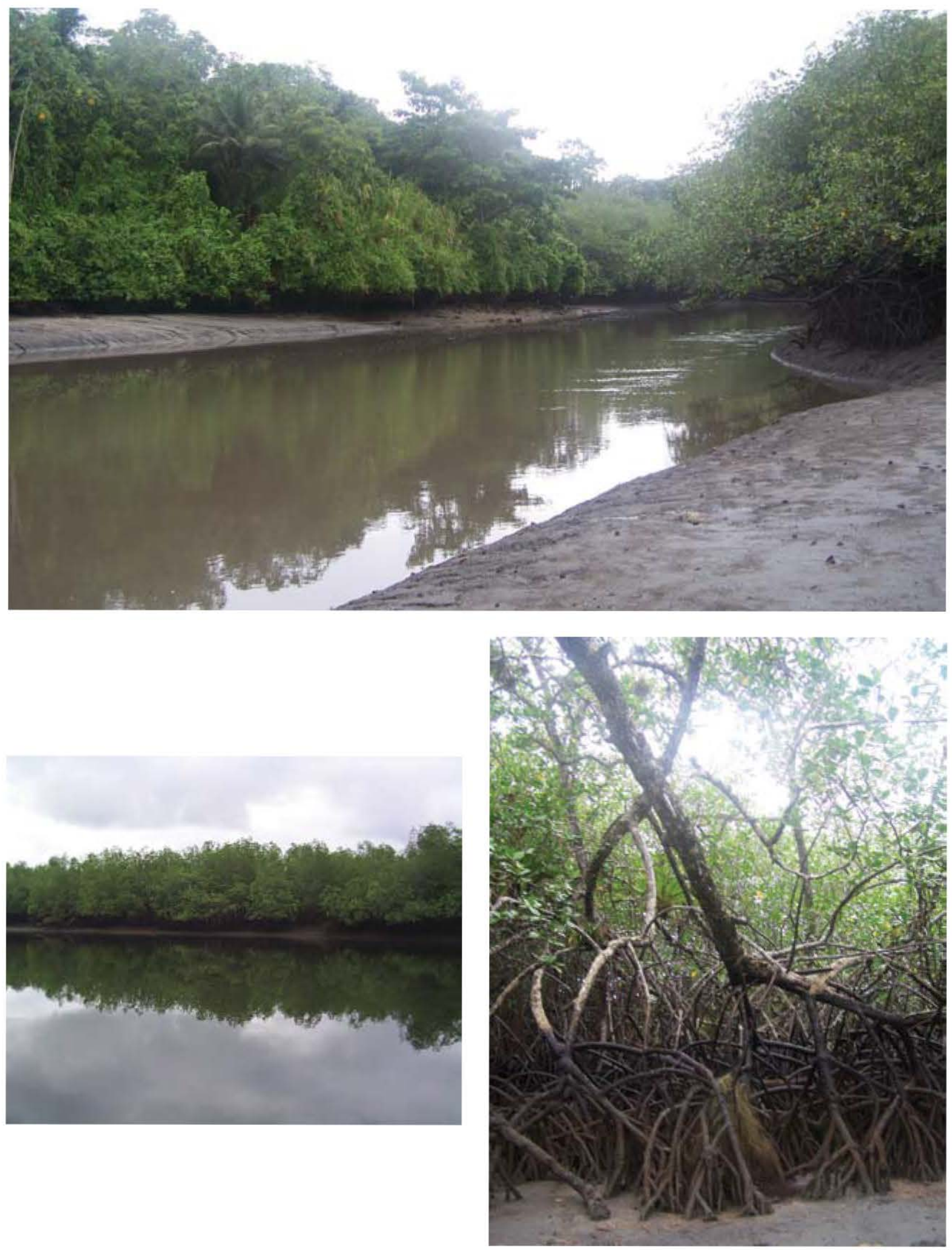

Figura 3. Bosque de manglar próximo al área de estudio. 

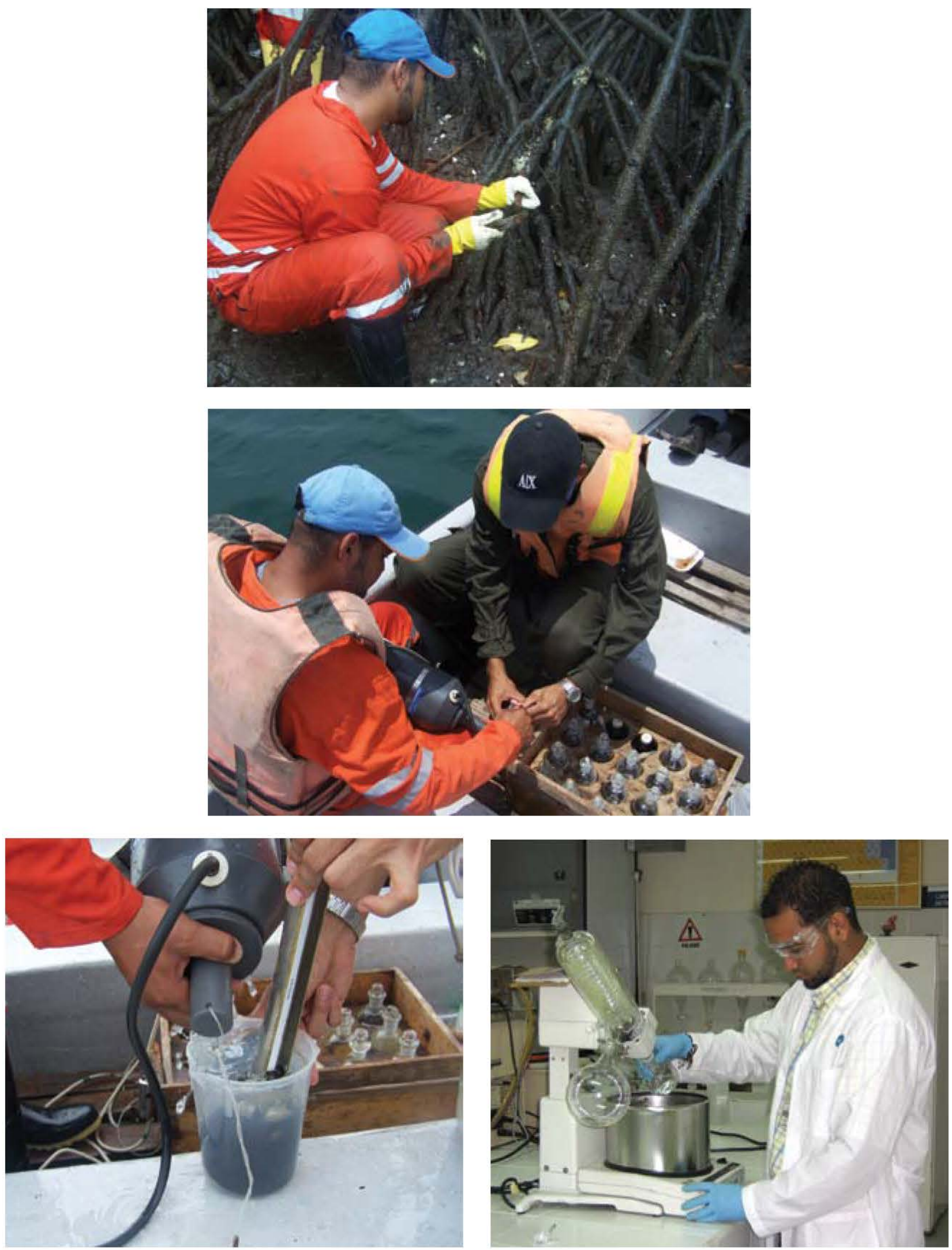

Figura 4. Trabajo en campo y análisis en laboratorio. 




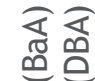
仓ิ 흔 들 응 बे 毞产 गु क्ष च 프 . 范

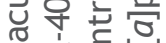
乐它的应 号要这 \& ป气 氕

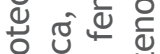
흔

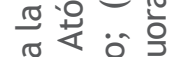

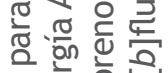
흐응


啳 중 ڤั \& 疋 응 巳 U. 웡

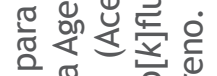
ข

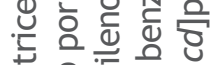
प유월

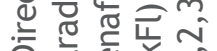
产造总 凹ैछ


满 ن 훤웡

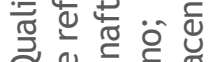
웡 兄.

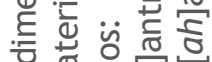



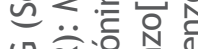



